Research Article

\title{
Integrated Sensor Fault Diagnosis and Fault-Tolerant Control for Manipulator
}

\author{
Bowen Hong, ${ }^{1}$ Lina Yao ${ }^{D},{ }^{1}$ and Zhiwei Gao ${ }^{2}{ }^{2}$ \\ ${ }^{1}$ School of Electrical Engineering, Zhengzhou University, Zhengzhou 450001, China \\ ${ }^{2}$ Faculty of Engineering and Environment, University of Northumbria, Newcastle upon Tyne NE2 8ST, UK
}

Correspondence should be addressed to Lina Yao; michelle_lnxq@126.com

Received 6 June 2019; Revised 3 September 2019; Accepted 6 September 2019; Published 29 September 2019

Academic Editor: Daniel Morinigo-Sotelo

Copyright (C) 2019 Bowen Hong et al. This is an open access article distributed under the Creative Commons Attribution License, which permits unrestricted use, distribution, and reproduction in any medium, provided the original work is properly cited.

\begin{abstract}
In this paper, an integrated scheme including fault diagnosis and fault-tolerant controller design is proposed for the manipulator system with the sensor fault. Any constant fault or time-varying fault can be estimated by the fault diagnosis scheme based on the adaptive observer rapidly and accurately, and the designed parameters can be solved by the linear matrix inequality. Using the fault estimation information, a fault-tolerant controller combining the characteristics of the proportional differentiation control and the sliding model control is designed to trace the expected trajectory via the back-stepping method. Finally, the effectiveness of the above scheme is verified by the simulation results.
\end{abstract}

\section{Introduction}

The security and reliability issues of the manipulator have been more and more attractive for the application of manipulators and increasingly widespread with the development of robotrelated research and industrial manufacturing. Generally, component damage and sensor and actuator failure will cause system faults, resulting in performance degradation. It has become an attractive and challenging problem to diagnose faults in time and design control schemes to reduce the impact of faults on the system. The research of the fault diagnosis and fault-tolerant control technology provides the theoretical basis for solving these problems in literature [1-4].

In the three categories of fault diagnosis theories, modelbased method, knowledge-based method, and signal processing-based method, the model-based fault diagnosis technology is popular with researchers [5]. In literature [6], based on the system model of the wind energy, an adaptive fault observer is constructed to detect and estimate the faults of the system, and this method also can be found in $[7,8]$. Literature [9] studies the fault model of the satellite actuators and uses the adaptive observer to estimate the actuator faults. A class of time-delayed nonlinear systems with the actuator fault and the uncertainties of the model parameter are considered in literature [10], and the adaptive fault diagnosis observer is designed for estimation of the nonlinear actuator fault. And more research can be found in [11-13].

During the past decade, the research on the fault diagnosis of manipulator system with actuator fault is preferred by researchers compared with the sensor fault, and the former research results are much more than those of the latter. For example, a sliding mode observer is constructed to diagnose the actuator fault in the manipulator in [14-16]. A new reconstruction method is developed for the actuator fault of the manipulator in [17] with the faster and more precise capability. The existing research on the sensor fault provides support for the next research in the manipulator system. For a class of nonlinear systems with sensor fault shown in $[18,19]$, the sensor fault is transformed as the actuator fault by a filter state; thus, the original system can be transformed into an augmented system with actuator fault, and then. the fault diagnosis observer is designed for the estimation of the fault. And in literature [20], the sensor faults in industrial robot manipulators are taken into account, and the fault is detected from the residual based on a bank of state observers via an analytical redundancy approach. 
Fault tolerance can be achieved passively or actively. Passive fault tolerance is robust with respect to possible system faults, but for the active fault tolerance, the obtained fault information is used to compensate for the influence of the fault $[5,21]$. After the fault information is available, the fault-tolerant controller can be designed to compensate for the fault impact. In literature $[22,23]$, the PID controller is introduced to compensate for the fault of the manipulator, which can be utilized to eliminate the impact of the fault. The manipulator fault system in literature $[24,25]$ can be secured to follow the desired trajectory with the design method of a sliding mode controller. The controller design strategy combining with the back-stepping method is introduced in literature $[26,27]$.

A lot of research has been done on fault diagnosis of the systems with actuator or sensor faults, but some of them are mainly concentrated on the manipulator systems with actuator faults, and others are mainly on the nonlinear systems with sensor faults. The manipulator system with sensor fault still lacks corresponding attention. Otherwise, when the adaptive observer is used to estimate the fault information in the manipulator system, it is unsatisfactory for the conventional scheme to estimate the fault, whether it is a slow or fast time-varying fault, even the constant fault. Therefore, it is motivated to make improvements for the fault diagnosis scheme so that the adaptive observer can be applied to the fault diagnosis of manipulator system with sensor fault.

The main attributions of this paper can be summarized as follows. The constraint condition of the conventional adaptive observer is modified, which enriches the design method of the adaptive observer; by modifying the condition, the fault diagnosis scheme proposed has the great performance whatever the constant or time-varying sensor fault; based on the sensor fault estimation information, the active fault-tolerant controller is designed to realize the target that the trajectory of the fault system can track the desired trajectory. This controller is combined with the outstanding characteristics of the PD control and the SMC technology via the back-stepping method.

The following contents are arranged as follows: In Chapter 2, the establishment of the state-space model of the manipulator sensor fault system is introduced and the main problem is described; the adaptive observer-based FD scheme is proposed to obtain the sensor fault information in Chapter 3; the fault-tolerant controller is designed with the fault information to track the desired trajectory in Chapter 4; Simulation results are given to verify the feasibility of the scheme and design in Chapter 5.

\section{Model Establishment}

When a sensor fault occurs, the dynamic model of the manipulator sensor fault system can be described as follows:

$$
\left\{\begin{array}{l}
M(q) \ddot{q}+C(q, \dot{q}) \dot{q}+g(q)=\tau+D_{d} d(t), \\
q_{\text {out }}=q+E_{s} f_{s}
\end{array}\right.
$$

where $q, \dot{q}$, and $\ddot{q}$ represent the angle, angular velocity, and angular acceleration, respectively, $\tau \in R^{n}$ is the control input, $M(q)$ is the inertia matrix, $C(q, \dot{q}) \dot{q}$ denotes the torques of the centripetal and Coriolis, and $g(q)$ denotes the gravitational torques. $q_{\text {out }}$ is defined as the output with the sensor fault information. $f_{s} \in R^{p}$ is the sensor fault.

And the norm of the sensor fault is satisfied $\left\|f_{s}(t)\right\| \leq f_{s \max }$ and $\left\|\dot{f}_{s}(t)\right\| \leq \alpha_{s} \leq \infty$, and its distribution matrix is denoted as $E_{s} \in R^{n \times p} . d(t) \in R^{p}$ is the disturbance, and $D_{d} \in R^{n \times p}$ denotes the disturbance distribution matrix.

In order to use the estimation information of the fault, the manipulator dynamic model will be transformed into the state-space model. Define the vector $x_{1}(t)=q \in R^{n}$ and $x_{2}(t)=\dot{q} \in R^{n}$, and the vector $x(t)=\left[\begin{array}{l}x_{1}(t) \\ x_{2}(t)\end{array}\right] \in R^{2 n}$, $u(t)=\tau \in R^{n}$, and $y(t) \in R^{2 n}$, and then the dynamic model of the manipulator sensor fault system (1) can be transformed into the state-space model [28]:

$$
\left\{\begin{array}{l}
\dot{x}(t)=A x(t)+B(x(t), u(t))+F(x(t))+D(x(t)) \\
y(t)=C x(t)+E f_{s}
\end{array}\right.
$$

where $\quad A=\left[\begin{array}{ll}0_{n} & I_{n} \\ 0_{n} & 0_{n}\end{array}\right], \quad B(x(t), u(t))=\left[\begin{array}{c}0_{n} \\ M\left(x_{1}\right)^{-1} u(t)\end{array}\right]$, $D(x(t), d)=\left[\begin{array}{c}0_{n} \\ M\left(x_{1}\right)^{-1} D_{d} d(t)\end{array}\right], \quad F(x(t))=$

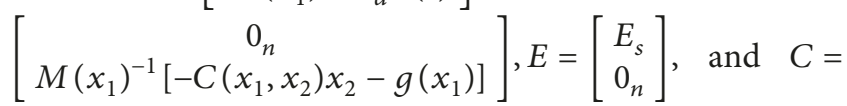
$\left[I_{2 n}\right]$.

For the convenience, it is necessary to introduce a new filter state to transform the fault diagnosis of the sensor fault to the fault diagnosis of the actuator fault. Here, the reconstruction variable is chosen as $[18,19,23]$

$$
\dot{x}_{s}(t)=-A_{s} x_{s}(t)+A_{s} y(t)
$$

where $-A_{s}$ is a Hurwitz matrix, and the new variable denotes as $\bar{x}(t)=\left[\begin{array}{c}x(t) \\ x_{s}(t)\end{array}\right] \in R^{4 n}$. Considering (2) and (3), the manipulator sensor fault augment system can be obtained as follows:

$$
\left\{\begin{aligned}
\dot{\bar{x}}(t)= & \bar{A} \bar{x}(t)+\bar{B}(\bar{x}(t), u(t))+\bar{F}(\bar{x}(t))+\bar{D}(\bar{x}(t), d) \\
& +\bar{E} f_{s}(t) \\
\bar{y}(t)= & \bar{C} \bar{x}(t)
\end{aligned}\right.
$$

where $\bar{A}=\left[\begin{array}{cc}I_{n} & 0_{n} \\ A_{n} C & -A_{n}\end{array}\right], \quad \bar{B}(\bar{x}(t), u)=\left[\begin{array}{c}B(x(t), u(t)) \\ 0_{2 n}\end{array}\right]$, $\bar{D}(\bar{x}(t), d)=\left[\begin{array}{c}D(x(t), d) \\ 0_{2 n}\end{array}\right], \quad \bar{F}(\bar{x}(t))=\left[\begin{array}{c}F(x(t)) \\ 0_{2 n}\end{array}\right], \quad \bar{C}=$ $\left[\begin{array}{ll}0_{2 n} & I_{2 n}\end{array}\right]$, and $\bar{E}=\left[\begin{array}{c}0_{2 n} \\ A_{s} E\end{array}\right]$.

Now, the sensor fault can be treated as the actuator fault in the augmented system, and the objective is to design an adaptive observer to obtain the fault estimation information. 


\section{Adaptive Observer-Based Fault Diagnosis Scheme}

The adaptive observer is designed as follows:

$$
\left\{\begin{aligned}
\dot{\bar{x}}(t)= & \bar{A} \hat{\bar{x}}(t)+\bar{B}(\hat{\bar{x}}(t), u)+\bar{F}(\hat{\bar{x}}(t))+\bar{E} \hat{f}_{s}(t) \\
& +L(\bar{y}(t)-\overline{\bar{y}}(t)) \\
\hat{\bar{y}}(t)= & \bar{C} \hat{\bar{x}}(t)
\end{aligned}\right.
$$

where $\bar{x}(t) \in R^{4 n}$ represents the estimation of the augmented state vector, $\hat{\bar{y}}(t)$ is the estimation of the augmented output vector, $\widehat{f}_{s}(t)$ is the estimation of the sensor fault, and $L \in R^{4 n \times 2 n}$ is the gain matrix of the observer.

Considering (4) and (5), defining the state error vector as $e_{x}(t)=\bar{x}(t)-\bar{x}(t)$, the system residual can be obtained as $e_{y}(t)=\bar{C}(\bar{x}(t)-\bar{x}(t))=\bar{C} e_{x}(t)$. The sensor fault estimation error is denoted as $e_{s}(t)=f_{s}(t)-\widehat{f}_{s}(t)$. Then, the state error dynamic system can be written as follows:

$$
\begin{aligned}
\dot{e}_{x}(t)= & \dot{\bar{x}}(t)-\dot{\overline{\bar{x}}}(t) \\
= & \bar{A} \bar{x}(t)+\bar{B}(\bar{x}(t), u)+\bar{F}(\bar{x}(t))+\bar{E} f_{s}(t) \\
& +\bar{D}(\bar{x}(t))-\bar{A} \hat{\bar{x}}(t)-\bar{B}(\hat{\bar{x}}(t), u)-\bar{F}(\hat{\bar{x}}(t)) \\
& -\bar{E} \widehat{f}_{s}(t)-L(\bar{y}(t)-\hat{\bar{y}}(t)) \\
= & (\bar{A}-L \bar{C}) e_{x}(t)+\bar{B}(\bar{x}(t), u)-\bar{B}(\widehat{\bar{x}}(t), u) \\
& +\bar{F}(\bar{x}(t))-\bar{F}(\hat{\bar{x}}(t))+\bar{D}(\bar{x}(t), d)+\bar{E} e_{s}(t) .
\end{aligned}
$$

Before introducing the main process of the FD scheme proposed in this paper, some significant lemma and assumptions are given as follows:

Lemma $1[19,29]$. There exists a scale $\mu>0$ making the inequality (7) be satisfied:

$$
2 a^{T} b \leq \frac{1}{\mu} a^{T} a+\mu b^{T} b, \quad a, b \in R^{n} .
$$

Assumption 1. It is assumed that for the manipulator sensor fault augmented system, the Lipschitz condition is satisfied. There exist the Lipschitz constants $\gamma_{1}$ and $\gamma_{2}$ making the inequality be satisfied:

$$
\begin{gathered}
\|\bar{B}(\bar{x}(t), u)-\bar{B}(\hat{\bar{x}}(t), u)\| \leq \gamma_{1}\|\bar{x}(t)-\hat{\bar{x}}(t)\|, \\
\|\bar{F}(\bar{x}(t))-\bar{F}(\hat{\bar{x}}(t))\| \leq \gamma_{2}\|\bar{x}(t)-\widehat{\bar{x}}(t)\| .
\end{gathered}
$$

Assumption 2. For the disturbance, the inequality $\| \bar{D}(\bar{x}(t)$, d) $\|\leq \bar{d}\| d(t) \|$ holds and $\bar{d}>0$.

Theorem 1. For the manipulator augment system, the above assumption holds. Given the scalars $M_{x}, M_{s}, M_{d}$, and $\rho$, there exist a positive definite symmetric matrix $P \in R^{4 n \times 4 n}$ and matrices $L \in R^{4 n \times 2 n}$ and $R_{s} \in R^{p \times 2 n}$ making the following conditions be satisfied:

$$
\begin{gathered}
\int e_{x}(t)^{T} M_{x} e_{x}(t) d s-\rho \int e_{s}(t)^{T} M_{s} e_{s}(t) d s \\
-\rho \int d(t)^{T} M_{d} d(t) d s \leq 0, \\
\Pi=\left[\begin{array}{ccc}
\Pi_{11} & \Pi_{12} & \Pi_{13} \\
* & \Pi_{22} & 0 \\
* & * & \Pi_{33}
\end{array}\right]<0,
\end{gathered}
$$

where $\Pi_{11}=\bar{A}^{T} P+P \bar{A}-\bar{C}^{T} Y^{T}-Y \bar{C}+2 \gamma P+M_{x}, \quad \Pi_{12}=$ $P \bar{E}-\bar{C}^{T} R_{s}^{T}, \quad \Pi_{13}=P \bar{d}, \quad \Pi_{22}=(1 / \mu)-\rho M_{s}, \quad \Pi_{33}=-\rho M_{d}$, and $Y=P L$, and * denotes the symmetric elements in $a$ symmetric matrix. The fault adaptive tuning rule

$$
\dot{\hat{f}}_{s}(t)=\Gamma_{s} R_{s} e_{y}(t)
$$

can realize that the $e_{x}(t)$ and $e_{s}(t)$ is uniformly ultimately bounded. $\Gamma_{s} \in R^{p \times p}$ is a prespecified matrix denoting the tuning rate, and $R_{s}$ is the gain matrix to be designed.

Remark 1. As is known to all, the constraint conditions in the theorem of the FD scheme based on the adaptive observer are usually formed as follows:

$$
\begin{aligned}
(\bar{A}-L \bar{C})^{T} P+P(\bar{A}-L \bar{C}) & <0, \\
\bar{E}^{T} P & =R_{s} \bar{C} .
\end{aligned}
$$

The fault adaptive tuning rule is shown as $\dot{\hat{f}}_{s}(t)=$ $\Gamma_{s} R_{s} e_{y}(t)$. The matrices $P, L, R_{s}$, and $\Gamma_{s}$ are defined as in Theorem 1. Its proof can be referred to [30] and is omitted here.

This conventional design of the above scheme is effective for various linear and nonlinear systems. However, when this scheme is used to estimate the sensor fault for the manipulator system, there are some issues to be considered: (1) because of the specific structure of the output matrix chosen in the augment system, it is easy to cause singularity when solving the positive definite symmetric matrix by LMI; (2) although it can diagnose the constant fault for various linear and nonlinear systems, it is unsatisfactory for the manipulator system of the time-varying fault, whether it is slow or fast varying, and even the constant fault.

Proof. Taking the Lyapunov function $V(t)=e_{x}^{T}(t) P e_{x}(t)+$ $e_{s}^{T}(t) \Gamma_{s}^{-1} e_{s}(t)$ into consideration, its first derivative with respect to time from (6) is formulated as follows:

$$
\begin{aligned}
\dot{V}(t)= & \dot{e}_{x}^{T}(t) P e_{x}(t)+e_{x}^{T}(t) P \dot{e}_{x}(t)+\dot{e}_{s}^{T}(t) \Gamma_{s}^{-1} e_{s}(t)+e_{s}^{T}(t) \Gamma_{s}^{-1} \dot{e}_{s}(t) \\
= & {\left[(\bar{A}-L \bar{C}) e_{x}(t)+\bar{B}(\bar{x}(t), u)-\bar{B}(\hat{\bar{x}}(t), u)+\bar{F}(\bar{x}(t))-\bar{F}(\widehat{\bar{x}}(t))+\bar{D}(\bar{x}(t))+\bar{E} e_{s}(t)\right]^{T} P e_{x}(t) } \\
& +e_{x}^{T}(t) P\left[(\bar{A}-L \bar{C}) e_{x}(t)+\bar{B}(\bar{x}(t), u)-\bar{B}(\overline{\bar{x}}(t), u)+\bar{F}(\bar{x}(t))-\bar{F}(\overline{\bar{x}}(t))+\bar{D}(\bar{x}(t), d)+\bar{E} e_{s}(t)\right] \\
& +2 e_{s}^{T}(t) \Gamma_{s}^{-1} \dot{e}_{s}(t) .
\end{aligned}
$$


According to Assumption 1, it can be obtained that

$$
\begin{aligned}
\dot{V}(t) \leq & e_{x}^{T}(t)\left[(\bar{A}-L \bar{C})^{T} P+P(\bar{A}-L \bar{C})+2 \gamma P\right] e_{x}(t) \\
& +2 e_{x}^{T}(t) P \bar{E} e_{s}(t)+2 e_{x}^{T}(t) P \bar{D}(x(t), d) \\
& +2 e_{s}^{T}(t) \Gamma_{s}^{-1} \dot{f}_{s}(t)-2 e_{s}^{T}(t) \Gamma_{s}^{-1} \dot{\hat{f}}_{s}(t) .
\end{aligned}
$$

According to Lemma 1, it can be shown that

$$
\begin{aligned}
e_{s}^{T}(t) \Gamma_{s}^{-1} \dot{f}_{s}(t) & \leq \frac{1}{\mu} e_{s}^{T}(t) e_{s}(t)+\mu \dot{f}_{s}^{T}(t) \Gamma_{s}^{-1 T} \Gamma_{s}^{-1} \dot{f}_{s}(t) \\
& \leq \frac{1}{\mu} e_{s}^{T}(t) e_{s}(t)+\delta_{s},
\end{aligned}
$$

where $\delta_{s}=\mu \alpha_{s}^{2} \lambda_{\max }\left(\Gamma_{s}^{-1} \Gamma_{s}^{-1}\right)$. And then equation (13) can be further written as follows:

$$
\begin{gathered}
\dot{V}(t) \leq e_{x}^{T}(t) Q e_{x}(t)+2 e_{x}^{T}(t) P \bar{E} e_{s}(t)+2 \bar{d} d^{T}(t) P e_{x}(t) \\
+\frac{1}{\mu} e_{s}^{T}(t) e_{s}(t)-2 e_{s}^{T}(t) R_{s} e_{y}(t)+\delta_{s},
\end{gathered}
$$

where $Q=(\bar{A}-L \bar{C})^{T} P+P(\bar{A}-L \bar{C})+2 \gamma P$. According to (9), the first derivative of $V(t)$ can be written as follows:

$$
\begin{gathered}
\dot{V}(t) \leq e_{x}^{T}(t) Q e_{x}(t)+2 e_{x}^{T}(t) P \bar{E} e_{s}(t)+2 \bar{d} d^{T}(t) P e_{x}(t) \\
+\frac{1}{\mu} e_{s}^{T}(t) e_{s}(t)-2 e_{x}^{T}(t) \bar{C}^{T} R_{s}^{T} e_{s}(t)+\delta_{s} .
\end{gathered}
$$

Define

$$
\begin{aligned}
H(t)= & \dot{V}(t)+e_{x}^{T}(t) M_{x} e_{x}(t)-\rho e_{s}^{T}(t) M_{s} e_{s}(t) \\
& -\rho d^{T}(t) M_{d} d(t) .
\end{aligned}
$$

Then, it can be obtained that

$$
\begin{aligned}
H(t) \leq & e_{x}^{T}(t) Q e_{x}(t)+e_{x}^{T}(t) M_{x} e_{x}(t)+2 e_{x}^{T}(t) P \bar{E} e_{s}(t) \\
& -2 e_{x}^{T}(t) \bar{C}^{T} R_{s}^{T} e_{s}(t)+\frac{1}{\mu} e_{s}^{T}(t) e_{s}(t)-\rho e_{s}^{T}(t) M_{s} e_{s}(t) \\
& +2 d^{T}(t) P \bar{d} e_{x}(t)-\rho d^{T}(t) M_{d} d(t)+\delta_{s} \\
\leq & e_{x}^{T}(t)\left(Q+M_{1}\right) e_{x}(t)+2 e_{x}^{T}(t)\left(P E-R_{s}^{T} \bar{C}^{T}\right) e_{s}(t) \\
& +e_{s}^{T}(t)\left(\frac{1}{\mu}-\rho M_{3}\right) e_{s}(t)+2 d^{T}(t) P \bar{d} e_{x}(t) \\
& -\rho d^{T}(t) M_{d} d(t)+\delta_{s} .
\end{aligned}
$$

Define a new state $\Lambda=\left[\begin{array}{lll}e_{x}^{T}(t) & e_{s}^{T}(t) & d^{T}(t)\end{array}\right]^{T}$. and from (9), it can be obtained that

$$
H(t) \leq \Lambda^{T} \Pi \Lambda+\delta .
$$

It can be further obtained that

$$
H(t) \leq-\pi\|\Lambda\|^{2}+\delta,
$$

where $\pi=\lambda_{\min }(-\Pi)$ and $\delta=\delta_{s}$. When $\pi\|\Lambda\|^{2}>\delta$ holds, it can be obtained that

$$
\dot{V}(t)+e_{x}^{T}(t) M_{x} e_{x}(t)-\rho e_{s}^{T}(t) M_{s} e_{s}(t)-\rho d^{T}(t) M_{d} d(t) \leq 0 .
$$

From (22) and (23), it can be indicated that under the Lyapunov stability theory, the new state $\Lambda$ is uniformly bounded and converges to a small set $\Psi=\left\{\Lambda \mid\|\Lambda\|^{2} \leq \delta / \pi\right\}$, which means that the state error vector $e_{x}(t)$ and the fault estimation error $e_{s}(t)$ are bounded and convergent.

\section{Fault-Tolerant Controller Design}

In order to obtain a good control performance, PID, PI, and $\mathrm{PD}$ controllers have been used for many systems. In recent years, sliding mode control (SMC) also has very good control effect for nonlinear systems. This paper combines the excellent characteristics of the PD controller and the SMC via back-stepping method to design the fault-tolerant controller. Because of the fault information severed by FD, the active FTC will be considered for the better control performance $[23,24,26]$.

When the sensor fault occurred in the manipulator system, the actual torque should be regarded as $q(t)=$ $q_{\text {out }}(t)-E_{s} f_{s}(t)$ as shown in (1). Once the fault is estimated, the actual torque can be expressed as follows:

$$
q(t)=q_{\text {out }}(t)-E_{s} \widehat{f}_{s}(t)
$$

Setting the desired torque trajectory as $q_{d}(t)$, then the tracking error can be obtained as

$$
e_{d}(t)=q(t)-q_{d}(t)=z_{1}(t) .
$$

The accessory controller is chosen as

$$
z_{2}(t)=\dot{z}_{1}(t)+c_{1} z_{1}(t),
$$

where $c_{1} \in R^{n \times n}$ is accessory controller parameter, and the $\mathrm{PD}$ controller is shown as

$$
u_{\mathrm{PID}}=K_{p} e_{d}+K_{d} \dot{e}_{d},
$$

where $K_{p} \in R^{n \times n}>0$ and $K_{d} \in R^{n \times n}>0$ is the proportional gain matrix and the differential gain matrix, respectively. Choose a sliding surface as

$$
s(t)=K_{p} e_{d}(t)+K_{d} \dot{e}_{d}(t)+z_{2}(t) .
$$

In order to shorten the reaching time and weaken the chattering phenomenon, the reaching law is chosen as the exponential type.

$$
\dot{s}(t)=-\alpha \operatorname{sign}(s(t))-\beta s(t),
$$

where $\alpha>0$ and $\beta>0$ are symmetric matrices, and $\beta$ should be much larger than $\alpha$. 


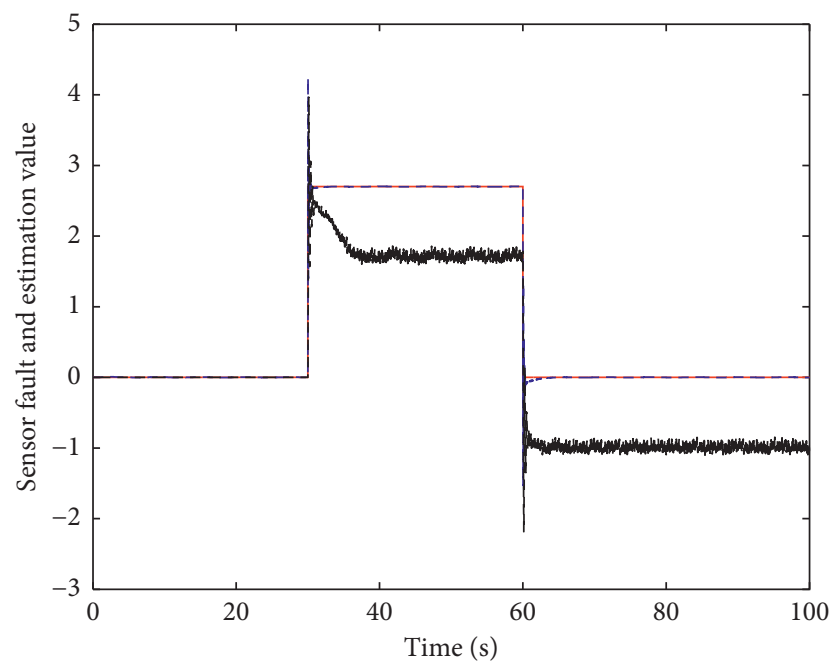

$\begin{array}{ll}\text { - Sensor fault } \\ \text { - - } & \text { Fault estimation 1 }\end{array}$

Figure 1: Constant fault and its estimation.

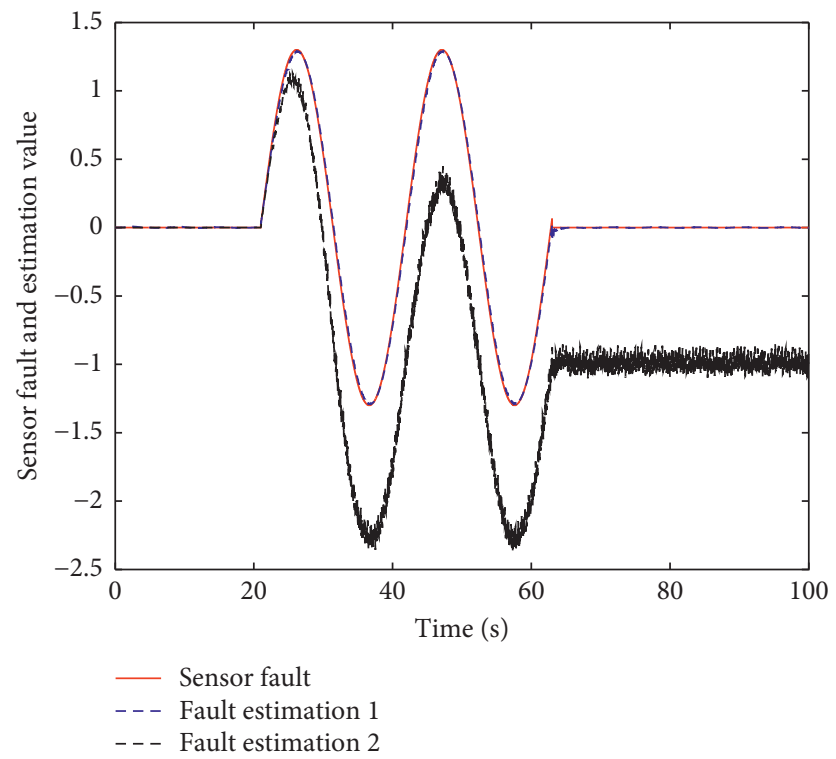

Figure 2: Slow time-varying fault and its estimation.

When it reaches to the sliding surface $\dot{s}=0$, the tracking error $e_{d} \longrightarrow 0$. The first derivative of the sliding surface (28) with respect to time is obtained as follows:

$$
\begin{aligned}
\dot{s}(t)= & K_{p} \dot{e}_{d}(t)+K_{d} \ddot{e}_{d}(t)+\dot{z}_{2}(t)=K_{p} \dot{z}_{1}(t)+K_{d} \ddot{z}_{1}(t)+\dot{z}_{2}(t) \\
= & K_{p}\left(z_{2}(t)-c_{1} z_{1}(t)\right)+K_{d} \ddot{z}_{1}(t)+\ddot{q}(t)-\ddot{q}_{d}(t)+c_{1} \dot{z}_{1}(t) \\
= & K_{p}\left(z_{2}(t)-c_{1} z_{1}(t)\right)+K_{d}\left\{M(q(t))^{-1}\left(u(t)+D_{d} d(t)\right)-\ddot{q}_{d}(t)-M(q(t))^{-1}[C(q(t), \dot{q}(t)) \dot{q}(t)\right. \\
& +g(q(t))]\}+M(q(t))^{-1}\left(u(t)+D_{d} d(t)\right)-\ddot{q}_{d}(t)+c_{1} \dot{z}_{1}(t)-M(q(t))^{-1}[C(q(t), \dot{q}(t)) \dot{q}(t)+g(q(t))] .
\end{aligned}
$$




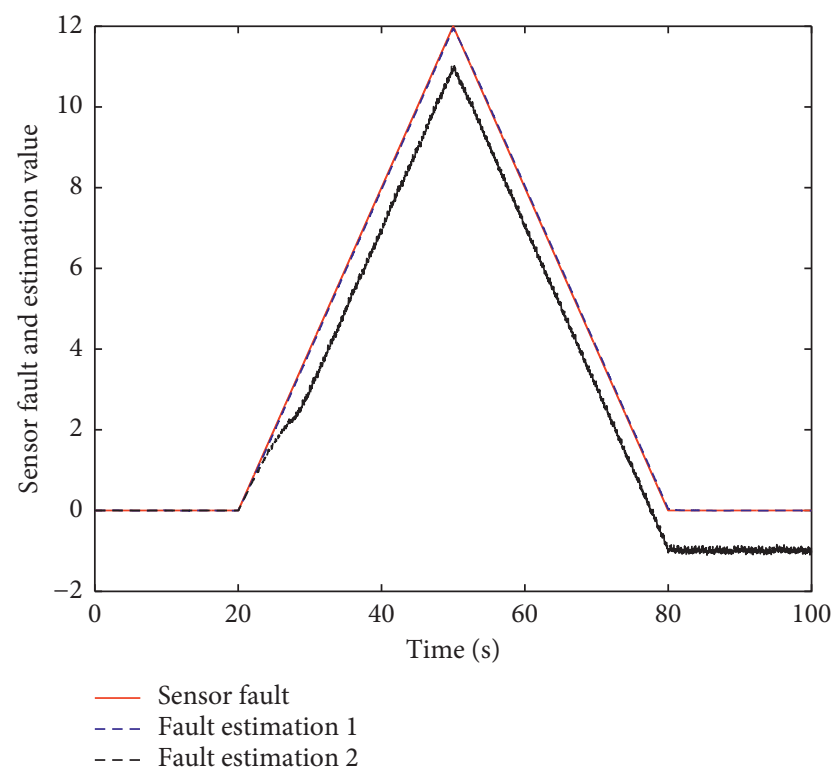

FIgURE 3: Fast time-varying fault and its estimation.

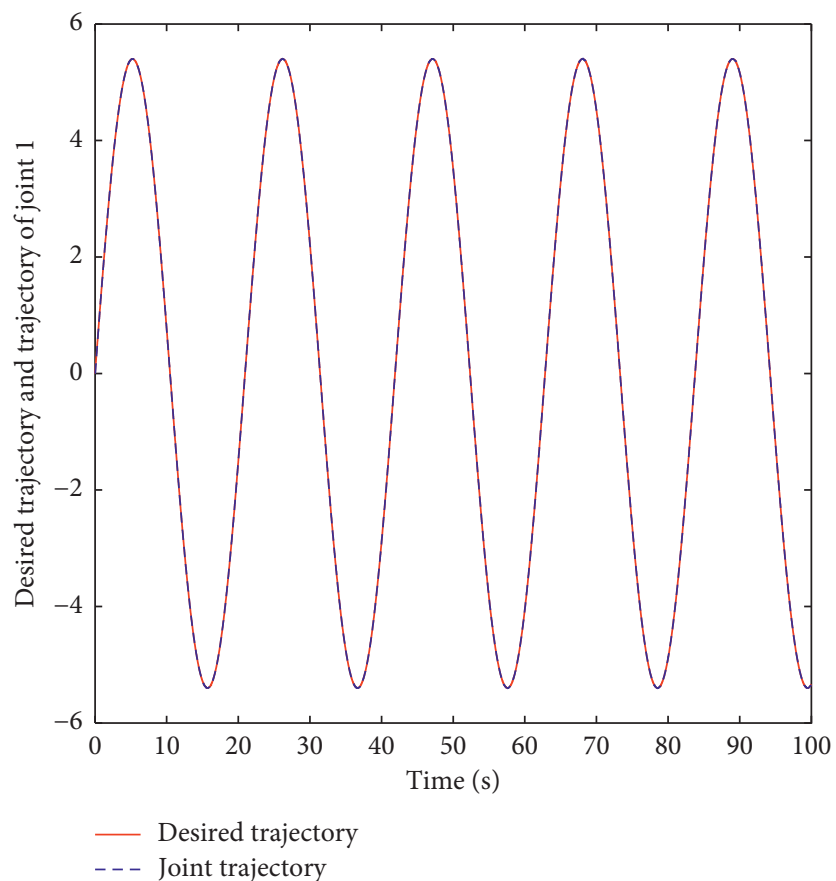

FIgURE 4: Desired trajectory and trajectory of joint 1.

According to (29) and (30), the fault-tolerant controller is designed as

$$
u_{c}(t)=u_{\mathrm{eq}}(t)+u_{d}(t)+u_{s}(t)
$$

$$
\begin{aligned}
u_{\mathrm{eq}}(t)= & M(q(t))\left\{-K_{p}\left(K_{d}+I\right)^{-1}\left(z_{2}(t)-c_{1} z_{1}(t)\right)\right. \\
& +M(q(t))^{-1}(C(q(t), \dot{q}(t)) \dot{q}(t)+g(q(t))) \\
& \left.+\ddot{q}_{d}(t)-\left(K_{d}+I\right)^{-1} c_{1} \dot{z}_{1}(t)-h\left(K_{d}+I\right)^{-1} s(t)\right\},
\end{aligned}
$$




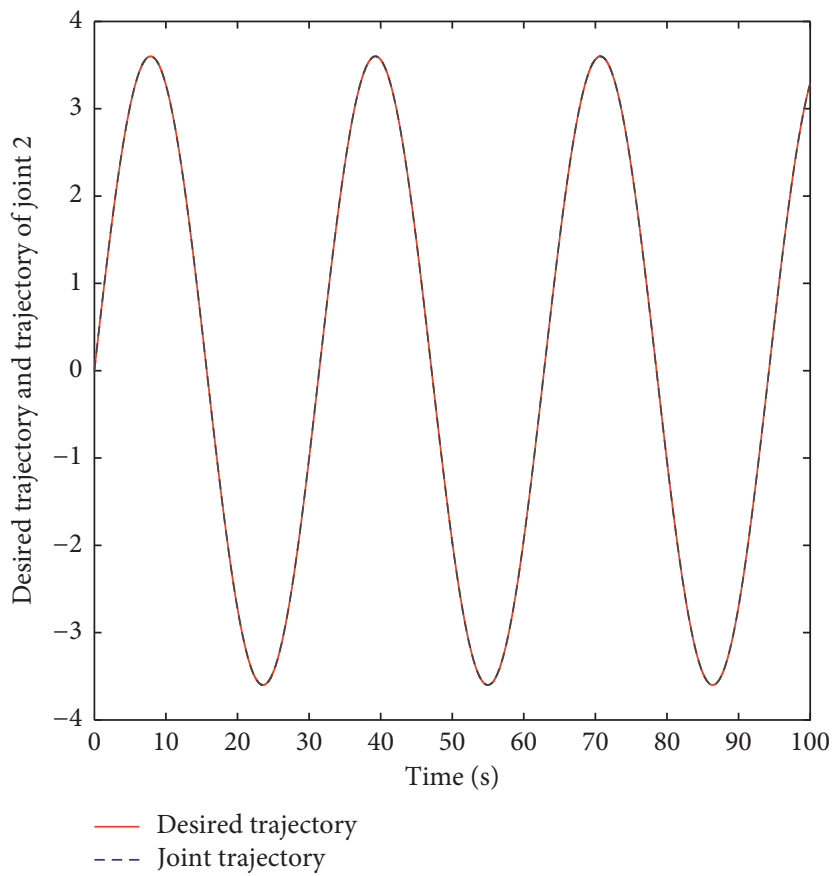

Figure 5: Desired trajectory and trajectory of joint 2.

where $h \in R^{n \times n}$ is an auxiliary parameter, and $I \in R^{n \times n}$ is the unit matrix. The disturbance compensation controller is designed as

$$
u_{d}(t)=-D_{d} d(t) \operatorname{sign}(s(t)),
$$

and, the reaching law controller is

$$
u_{s}(t)=-M(q(t))\left(K_{d}+I\right)^{-1}(\alpha \operatorname{sign}(s(t))+\beta s(t)) .
$$

Proof. Taking the Lyapunov function $V_{c}(t)=(1 / 2) s^{T}(t)$ $s(t)+(1 / 2) z_{1}^{T}(t) z_{1}(t)$ into consideration, its first derivative with respect to time is obtained as follows:

$$
\begin{aligned}
\dot{V}_{c}(t) & =s^{T}(t) \dot{s}(t)+z_{1}^{T}(t) \dot{z}_{1}(t) \\
& =z_{1}^{T}(t) z_{2}(t)-z_{1}^{T}(t) c_{1} z_{1}(t)+s^{T}(t) \dot{s}(t) .
\end{aligned}
$$

By substituting (30) into equation (35), it can be obtained that

$$
\begin{aligned}
\dot{V}_{c}(t)= & z_{1}^{T}(t) z_{2}(t)-z_{1}^{T}(t) c_{1} z_{1}(t)+s^{T}(t)\left\{K _ { p } \left(z_{2}(t)\right.\right. \\
& \left.-c_{1} z_{1}(t)\right)+K_{d}\left(M(q(t))^{-1}\left(u(t)+D_{d} d(t)\right)\right. \\
& \left.-M(q(t))^{-1}[C(q(t), \dot{q}(t))+g(q(t))]-\ddot{q}_{d}(t)\right) \\
& -M(q(t))^{-1}[C(q(t), \dot{q}(t)) \dot{q}(t)+g(q(t))] \\
& \left.+M(q(t))^{-1}\left(u(t)+D_{d} d(t)\right)-\ddot{q}_{d}(t)+c_{1} \dot{z}_{1}(t)\right\} .
\end{aligned}
$$

Substituting (31) into (36), it can be obtained that

$$
\begin{aligned}
\dot{V}_{c}(t)= & z_{1}^{T}(t) z_{2}(t)-z_{1}^{T}(t) c_{1} z_{1}(t)+s^{T}(t)\left\{K _ { p } \left(z_{2}(t)\right.\right. \\
& \left.-c_{1} z_{1}(t)\right)+K_{d}\left(M ( q ( t ) ) ^ { - 1 } \left(u_{\mathrm{eq}}(t)+u_{d}(t)\right.\right. \\
& \left.+u_{s}(t)+D_{d} d(t)\right)-M(q(t))^{-1} \\
& \left.\cdot[C(q(t), \dot{q}(t)) \dot{q}(t)+g(q(t))]-\ddot{q}_{d}(t)\right) \\
& +M(q(t))^{-1}\left(u(t)+D_{d} d(t)\right)-M(q(t))^{-1} \\
& \left.\cdot[C(q(t), \dot{q}(t)) \dot{q}(t)+g(q(t))]-\ddot{q}_{d}(t)+c_{1} \dot{z}_{1}(t)\right\} .
\end{aligned}
$$

Then it can be further formulated that

$$
\begin{aligned}
\dot{V}_{c}(t)= & z_{1}^{T}(t) z_{2}(t)-z_{1}^{T}(t) c_{1} z_{1}(t)-s^{T}(t) h s(t) \\
& -\alpha s^{T}(t) \operatorname{sign}(s(t))-\beta s^{T}(t) s(t) \\
= & -Z^{T}(t) \Phi Z(t)-\alpha\left\|s^{T}(t)\right\|-\beta\left\|s^{T}(t) s(t)\right\| \\
= & -\phi\left\|Z^{T}(t) Z(t)\right\|-\alpha\left\|s^{T}(t)\right\|-\beta\left\|s^{T}(t) s(t)\right\|,
\end{aligned}
$$

where the state vector $Z(t)=\left[\begin{array}{ll}z_{1}^{T}(t) & z_{2}^{T}(t)\end{array}\right]^{T}, \quad \Phi=$ $\left[\begin{array}{ll}\Phi_{11} & \Phi_{12} \\ \Phi_{21} & \Phi_{22}\end{array}\right]$, and $\phi=\lambda_{\min }(\Phi)$ represents the minimum eigenvalue of $\Phi$. And $\Phi_{11}, \Phi_{12}, \Phi_{21}$, and $\Phi_{22}$ can be given as follows: $\Phi_{11}=c_{1}+K_{p}^{T} h K_{p}-K_{p}^{T} h K_{d} c_{1}-c_{1}^{T} K_{d} h K_{p}+c_{1}^{T} K_{d} h$ $K_{d} c_{1}, \Phi_{21}=\Phi_{12}^{T}, \Phi_{12}=K_{p}^{T} h K_{d}+K_{p}^{T} h-c_{1}^{T} K_{d} h K_{d}-c_{1}^{T} K_{d}$ $h-(1 / 2) I$, and $\Phi_{22}=h+h K_{d}+K_{d}^{T} h+K_{d}^{T} h K_{d}$.

The inequality $\dot{V}_{c}(t) \leq 0$ will be satisfied if and only if $c_{1}$, $K_{p}, K_{d}$, and $h$ are selected to make $\Phi>0$ be ensured 
appropriately, which means the tracking error is converged under the fault-tolerant controller.
5.1. The Simulation for FD Scheme. The coefficient matrix of the manipulator model is given as

\section{Computer Simulation Results}

In order to verify the effectiveness of the proposed fault diagnosis scheme and fault-tolerant control algorithm, a simulation experiment is carried out.

$$
\begin{aligned}
M(q) & =\left(\begin{array}{cc}
\left(m_{1}+m_{2}\right) l_{1}^{2}+m_{2} l_{2}^{2}+2 m_{2} l_{1} l_{2} \cos \left(q_{2}\right) & m_{2}\left(l_{2}^{2}+l_{1} l_{2} \cos \left(q_{2}\right)\right) \\
m_{2}\left(l_{2}^{2}+l_{1} l_{2} \cos \left(q_{2}\right)\right) & m_{2} l_{2}^{2}
\end{array}\right), \\
C(q, \dot{q}) \dot{q} & =\left(\begin{array}{c}
-2 m_{2} l_{1} l_{2} \sin \left(q_{2}\right) \dot{q}_{1} \dot{q}_{2}-m_{2} l_{1} l_{2} \sin \left(q_{2}\right) \dot{q}_{2}^{2} \\
m_{2} l_{1} l_{2} \sin \left(q_{2}\right) \dot{q}_{2}^{2}
\end{array}\right), \\
g(q) & =\left(\begin{array}{c}
\left(m_{1}+m_{2}\right) l_{1} g \cos \left(q_{1}\right)+m_{2} l_{2} g \cos \left(q_{1}+q_{2}\right) \\
m_{2} l_{2} g \cos \left(q_{1}+q_{2}\right)
\end{array}\right),
\end{aligned}
$$

where $l_{1}=1 \mathrm{~m}, l_{2}=0.8 \mathrm{~m}$, and $m_{1}=m_{2}=1 \mathrm{~kg}$. The fault distribution matrix and the disturbance distribution matrix are $E_{s}=\left[\begin{array}{l}1 \\ 1\end{array}\right]$ and $D_{d}=\left[\begin{array}{l}1 \\ 1\end{array}\right]$, respectively. The scalars of the observer are given as $M_{x}=1.5, M_{s}=3.5$, $M_{d}=5, \rho=2.5$, and $\Gamma_{s}=15$. Choosing $\mu=1$, it can be obtained that

$$
L=\left[\begin{array}{rrrr}
384.3025 & 291.3311 & 12.7349 & -14.9555 \\
291.3013 & 384.3099 & -14.9555 & 12.7411 \\
9.1632 & -5.9876 & 88.4862 & 0.2886 \\
-5.9878 & 9.1574 & 0.2893 & 88.4865 \\
19.3345 & 3.6953 & 0.7730 & -0.8442 \\
3.6919 & 19.3342 & -0.8439 & 0.7737 \\
0.6257 & 0.2252 & 15.2663 & 0.0862 \\
0.2254 & 0.6249 & 0.0862 & 15.2663
\end{array}\right],
$$

The FD scheme will be experimented in three cases with different fault types. Case 1 is a constant fault, and the fault occurs between $30 \mathrm{~s}$ and $60 \mathrm{~s}$; Case 2 is a sinusoidal slow time-varying fault when the time interval is from $26 \mathrm{~s}$ to $73 \mathrm{~s}$; on the contrary, Case 3 is selected as the fast timevarying fault in $20 \mathrm{~s}$ to $80 \mathrm{~s}$. The details are shown as follows.

Case 1: constant fault $f_{s}(t)= \begin{cases}2.7, & t \in[30,60], \\ 0, & \text { else }\end{cases}$

$$
\begin{aligned}
& \text { Case 2: slow time-varying fault } f_{s}(t)= \\
& \begin{cases}1.3 \sin (0.3 t), \quad t \in[21,63], \\
0, & \text { else, }\end{cases} \\
& \text { Case } 3: \text { fast time-varying fault } f_{s}(t)= \\
& \begin{cases}0.4(t-20), & t \in[20,50], \\
24-0.4(t-20), & t \in[50,80], \\
0, & \text { else, }\end{cases}
\end{aligned}
$$

As shown in the figures, the fault estimation 1 is the estimation of the sensor fault by the scheme proposed in this paper, and the fault estimation 2 is estimated by the conventional scheme. Figure 1 gives the estimation of the constant fault. It can be observed that the response speed is very fast, and the fault value can be accurately estimated. In addition, the advantage of the fast response is also reflected in the Case 2 and 3 shown in Figure 2 and Figure 3, respectively. In Figure 2, it can be found that although the fault changes with time, the observer can estimate the fault information accurately. Figure 3 depicts the fast time-varying fault and its estimation, and its accuracy is as good as Case 1 and Case 2. But, the conventional scheme can not meet the accuracy and speed requirements.

5.2. The Simulation for FTC. Take the Case 3 as the example to verify the effect of the active fault-tolerant controller designed, and the desired trajectory is specifically set to $q_{d}(t)=\left[\begin{array}{l}5.4 \sin (0.3 t) \\ 3.6 \sin (0.2 t)\end{array}\right]$. The parameters designed are as given follows: $c_{1}=\left[\begin{array}{ll}1 & 0 \\ 0 & 1\end{array}\right], h=\left[\begin{array}{ll}1 & 0 \\ 0 & 1\end{array}\right], K_{p}=\left[\begin{array}{cc}100 & 0 \\ 0 & 100\end{array}\right]$, $K_{d}=\left[\begin{array}{ll}2 & 0 \\ 0 & 2\end{array}\right], \alpha=\left[\begin{array}{ll}5 & 0 \\ 0 & 5\end{array}\right]$, and $\beta=\left[\begin{array}{cc}20 & 0 \\ 0 & 25\end{array}\right]$.

From Figures 4 and 5, the each joint trajectory can follow the desired trajectory well with the fault-tolerant 
controller, and the influence of the sensor fault can be eliminated, which means that the fault-tolerant controller designed here makes sense for the manipulator sensor fault system.

\section{Conclusions}

In this paper, the integrated fault diagnosis and faulttolerant control scheme is proposed for the sensor fault in the manipulator system. By modifying the existing condition of the adaptive observer, any constant fault or timevarying fault can be estimated by the fault diagnosis scheme based on the adaptive observer rapidly and accurately, and the designed parameters can be solved by the LMI. In addition, the designed fault-tolerant controller combines the excellent characteristics of the PD controller and the sliding mode controller via the back-stepping method, performing a good effect when the sensor occurs in the manipulator system. The validity of the scheme and design is verified by the simulation experiments on a manipulator model. For the further research, the fault diagnosis and fault-tolerant control scheme would be designed when a fault occurs considering time-delay or uncertainty in the manipulator system. At this point, not only the impact of the fault to be considered but also the time-delay or the uncertain disturbance will be taken into consideration to ensure the stability of the system performance, which will be challenging but more practical.

\section{Data Availability}

The data used to support the findings of this study are available from the corresponding author upon request.

\section{Conflicts of Interest}

The authors declare that they have no conflicts of interest.

\section{References}

[1] R. Isermann, "Model-based fault-detection and diagnosisstatus and applications," Annual Reviews in Control, vol. 29, no. 1, pp. 71-85, 2005.

[2] J. Zaytoon and S. Lafortune, "Overview of fault diagnosis methods for discrete event systems," Annual Reviews in Control, vol. 37, no. 2, pp. 308-320, 2013.

[3] Z. Gao, S. X. Ding, and C. Cecati, "Real-time fault diagnosis and fault-tolerant control," IEEE Transactions on Industrial Electronics, vol. 62, no. 6, pp. 3752-3756, 2015.

[4] R. J. Patton, "Fault-tolerant control: the 1997 situation," IFAC Proceedings Volumes, vol. 30, no. 18, pp. 1029-1051, 1997.

[5] Z. Gao, C. Cecati, and S. X. Ding, "A survey of fault diagnosis and fault-tolerant techniques-part I: fault diagnosis with model-based and signal-based approaches," IEEE Transactions on Industrial Electronics, vol. 62, no. 6, pp. 3757-3767, 2015.

[6] Z.-Q. Wu, Y. Yang, and C.-H. Xu, "Adaptive fault diagnosis and active tolerant control for wind energy conversion system," International Journal of Control, Automation and Systems, vol. 13, no. 1, pp. 120-125, 2015.
[7] P. M. Frank, S. X. Ding, and T. Marcu, "Model-based fault diagnosis in technical processes," Transactions of the Institute of Measurement and Control, vol. 22, no. 1, pp. 57-101, 2000.

[8] W. Chen and M. Saif, "Observer-based strategies for actuator fault detection, isolation and estimation for certain class of uncertain nonlinear systems," IET Control Theory \& Applications, vol. 1, no. 6, pp. 1672-1680, 2007.

[9] Z. Wang, "Nonlinear adaptive observer-based fault diagnosis for satellite actuator," Applied Mechanics and Materials, vol. 214, pp. 851-855, 2012.

[10] L. Yao, H. Wang, and V. Cocquempot, "Adaptive nonlinear actuator fault diagnosis for uncertain nonlinear systems with time-varying delays," Asian Journal of Control, vol. 16, no. 4, pp. 1057-1065, 2014.

[11] L. Cao and B. Xiao, Exponential and Resilient Control for Attitude Tracking Maneuvering of Spacecraft with Actuator Uncertainties, IEEE/ASME Transactions on Mechatronics, Piscataway, NJ, USA, 2019.

[12] B. Xiao, X. Yang, H. R. Karimi, and J. Qiu, "Asymptotic tracking control for a more representative class of uncertain nonlinear systems with mismatched uncertainties," IEEE Transactions on Industrial Electronics, vol. 66, no. 12, 2019.

[13] L. Cao, D. Ran, X. Chen, X. Li, and B. Xiao, "Huber secondorder variable structure predictive filter for satellites attitude estimation," International Journal of Control, Automation and Systems, vol. 17, no. 7, pp. 1781-1792, 2019.

[14] F. Zhu and J. Yang, "Fault detection and isolation design for uncertain nonlinear systems based on full-order, reducedorder and high-order high-gain sliding-mode observers," International Journal of Control, vol. 86, no. 10, pp. 1800-1812, 2013.

[15] L. M. Capisani, A. Ferrara, A. Ferreira de Loza, and L. M. Fridman, "Manipulator fault diagnosis via higher order sliding-mode observers," IEEE Transactions on Industrial Electronics, vol. 59, no. 10, pp. 3979-3986, 2012.

[16] D. Brambilla, L. M. Capisani, A. Ferrara, and P. Pisu, "Fault detection for robot manipulators via second-order sliding modes," IEEE Transactions on Industrial Electronics, vol. 55, no. 11, pp. 3954-3963, 2008.

[17] B. Xiao and S. Yin, "An intelligent actuator fault reconstruction scheme for robotic manipulators," IEEE Transactions on Cybernetics, vol. 48, no. 2, pp. 639-647, 2018.

[18] Q. Jia, W. Chen, Y. Zhang, and H. Li, "Fault reconstruction for continuous-time systems via learning observers," Asian Journal of Control, vol. 18, no. 2, pp. 549-561, 2016.

[19] K. Zhang, B. Jiang, P. Shi, and V. Cocquempot, "Adaptive observer-based fast fault estimation," International Journal of Control, Automation, and Systems, vol. 6, no. 3, pp. 320-326, 2008.

[20] G. Paviglianiti, F. Pierri, F. Caccavale, and M. Mattei, "Robust fault detection and isolation for proprioceptive sensors of robot manipulators," Mechatronics, vol. 20, no. 1, pp. 162-170, 2010.

[21] J. Jiang and X. Yu, "Fault-tolerant control systems: a comparative study between active and passive approaches," $A n$ nual Reviews in Control, vol. 36, no. 1, pp. 60-72, 2012.

[22] W. Yu and J. Rosen, "Neural PID control of robot manipulators with application to an upper limb exoskeleton," IEEE Transactions on Cybernetics, vol. 43, no. 2, pp. 673-684, 2013.

[23] V. Parra-Vega, S. Arimoto, Y. H. Liu, G. Hirzinger, and P. Akella, "Dynamic sliding PID control for tracking of robot manipulators: theory and experiments," IEEE Transactions on Robotics and Automation, vol. 19, no. 6, pp. 967-976, 2003. 
[24] W. Zheng and M. Chen, "Tracking control of manipulator based on high-order disturbance observer," IEEE Access, vol. 6, pp. 26753-26764, 2018.

[25] M. Zeinali and L. Notash, "Adaptive sliding mode control with uncertainty estimator for robot manipulators," Mechanism and Machine Theory, vol. 45, no. 1, pp. 80-90, 2010.

[26] M. Van, M. Mavrovouniotis, and S. S. Ge, "An adaptive backstepping nonsingular fast terminal sliding mode control for robust fault tolerant control of robot manipulators," IEEE Transactions on Systems, Man, and Cybernetics: Systems, vol. 49, no. 7, pp. 1448-1458, 2018.

[27] M. Van, H.-J. Kang, and K.-S. Shin, "Backstepping quasicontinuous high-order sliding mode control for a TakagiSugeno fuzzy system with an application for a two-link robot control," Proceedings of the Institution of Mechanical Engineers, Part C: Journal of Mechanical Engineering Science, vol. 228, no. 9, pp. 1488-1500, 2014.

[28] H.-J. Ma and G.-H. Yang, "Simultaneous fault diagnosis for robot manipulators with actuator and sensor faults," Information Sciences, vol. 366, pp. 12-30, 2016.

[29] D. Kharrat, H. Gassara, A. El Hajjaji, and M. Chaabane, "Adaptive observer and fault tolerant control for takagisugeno descriptor nonlinear systems with sensor and actuator faults," International Journal of Control, Automation and Systems, vol. 16, no. 3, pp. 972-982, 2018.

[30] B. Jiang, M. Staroswiecki, and V. Cocquempot, "Fault accommodation for nonlinear dynamic systems," IEEE Transactions on Automatic Control, vol. 51, no. 9, pp. 1578-1583, 2006. 


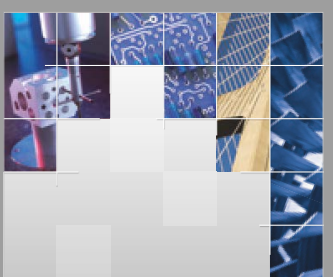

\section{Enfincering}
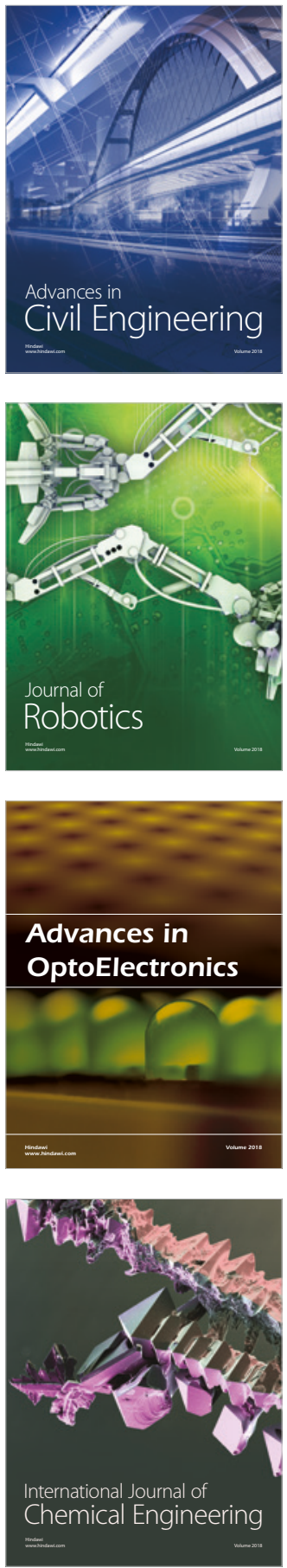

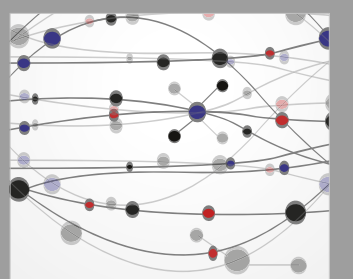

\section{Rotating \\ Machinery}

The Scientific World Journal

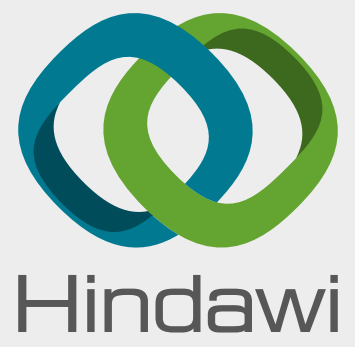

Submit your manuscripts at

www.hindawi.com
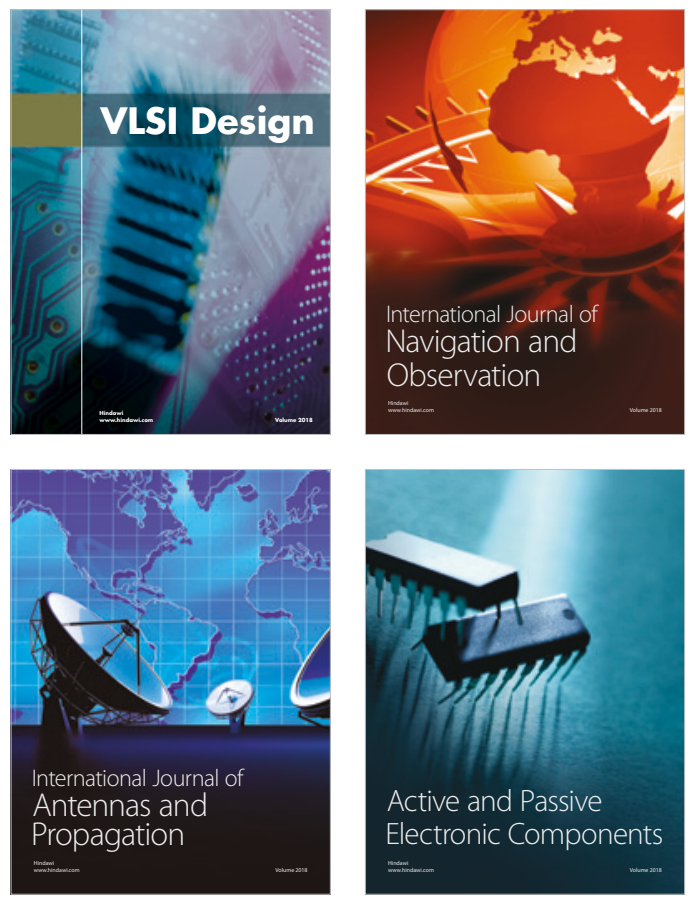
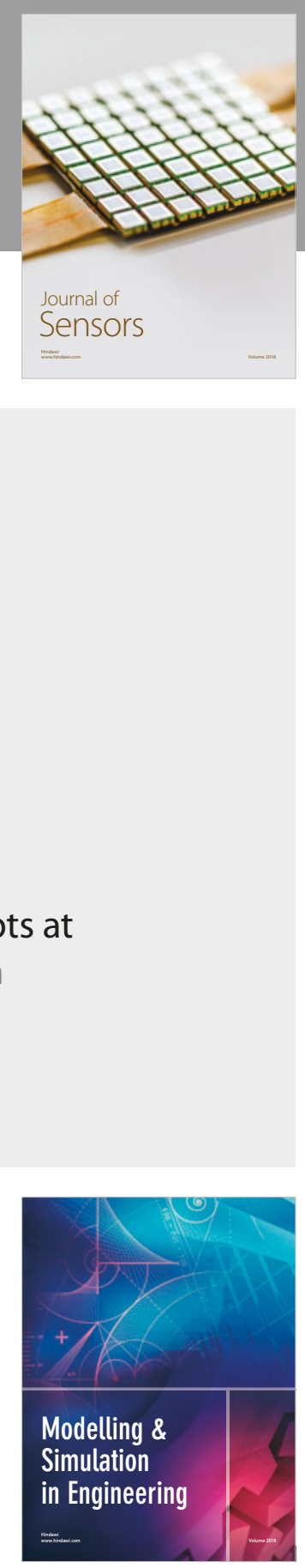

\section{Advances \\ Multimedia}
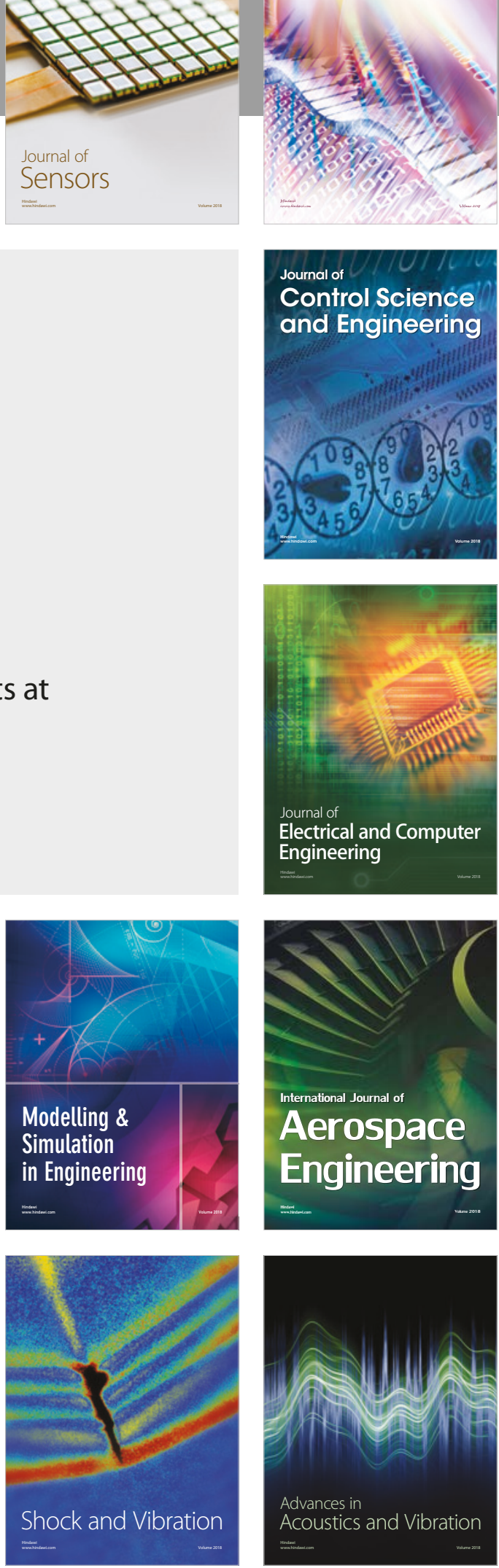